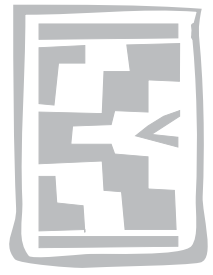

\title{
Red blood cell volume as a predictor of fatal reactions in cattle infected with Theileria parva Katete
}

\author{
P. FANDAMU ${ }^{1}$, T. MARCOTTY ${ }^{2}$, J.R.A. BRANDT ${ }^{2}$, L. DUCHATEAU ${ }^{3}$, N. SPEYBROECK ${ }^{2}$, \\ T.T. DOLAN ${ }^{4}{ }^{\dagger}$ and D. BERKVENS ${ }^{2 *}$
}

\begin{abstract}
FANDAMU, P., MARCOTTY, T., BRANDT, J.R.A., DUCHATEAU, L., SPEYBROECK, N., DOLAN, T.T. \& BERKVENS, D. 2007. Red blood cell volume as a predictor of fatal reactions in cattle infected with Theileria parva Katete. Onderstepoort Journal of Veterinary Research, 74:37-43

A comparison of mean corpuscular volume (MCV) and packed cell volume (PCV) was made between cattle undergoing lethal and non-lethal reactions following experimental infections with the apicomplexan protozoa, Theileria parva Katete. This work confirmed that anaemia occurs in infected animals. However, the fall in PCV was steeper in lethal reactions compared to non-lethal reactions. Our results show that animals with initially lower MCV values are more prone to fatal reaction, despite having normal PCV profiles. The study also found that small red blood cells are more likely to be infected with T. parva. These findings suggest that animals with a higher proportion of small red blood cells in circulation will be more likely to succumb to $T$. parva infections. The potential for using MCV as a predictor of the outcome of infection challenge is discussed.
\end{abstract}

Keywords: Anaemia, cattle, mean corpuscular volume, packed cell volume, prediction of outcome, Theileria parva, Zambia

\section{INTRODUCTION}

Theileria parva is a tick-borne protozoan parasite of cattle that causes an acute, often fatal lymphoproliferative disease known as East Coast fever (ECF). Theileria parva multiplies in bovine lymphocytes by

* Author to whom correspondence is to be directed. E-mail: dberkvens@itg.be

+ Deceased 15 January 2007

1 Department of Veterinary and Livestock Development, Mazabuka Veterinary Research Station, P.O. Box 670050, Mazabuka, Zambia

2 Department of Animal Health, Institute of Tropical Medicine, Nationalestraat 155, B-2000 Antwerpen, Belgium

3 Department of Physiology, Biochemistry and Biometrics, Faculty of Veterinary Medicine, Ghent University, Salisburylaan 133, B-9820, Belgium

4 Livestock Services Limited, P.O. Box 24437, 00502 Karen, Nairobi, Kenya

Accepted for publication 28 September 2006-Editor schizogony resulting in the production of merozoites that invade red blood cells (RBCs) and develop into piroplasms (Conrad, Denham \& Brown 1986). The major clinical signs in $T$. parva infections are enlargement of lymph nodes, especially those adjacent to sites of parasite inoculation, pyrexia, lymphocyte destruction resulting in leukopenia, interstitial pneumonia, pulmonary oedema and death (Jura \& Losos 1980; Irvin \& Mwamachi 1983; Irvin \& Morrison 1987). Other signs include blood-tinged diarrhoea, lacrimation and corneal opacity, splenic enlargement and lymphomata in the kidneys. Animals usually die within 30 days after infection (Irvin \& Morrison 1987). Some animals die within 24 days as a result of respiratory distress while others die later following secondary infections and wasting (Irvin \& Mwamachi 1983; Irvin \& Morrison 1987). Anaemia has not been recognised as a major feature in classical $T$. parva infections (Maxie, Dolan, Jura, Tabel \& Flowers 1982) but it has been described in cattle infected with 
certain stocks of $T$. parva (Norval, Perry \& Young 1992; Mbassa, Balemba, Maselle \& Mwaga 1994). The pathogenesis of the anaemia in T. parva infections is not well understood (Mbassa et al. 1994). In infections with the related Theileria annulata and Theileria mutans parasites, anaemia is common and is attributed to the replication of the parasite in the erythrocytes (Young, Purnell, Payne, Brown \& Kanhai 1978; Norval et al. 1992). The severity of T. parva infections has been linked to the quantity of the infective dose (Radley, Brown, Burridge, Cunningham, Peirce \& Purnell 1974; Dolan, Young, Losos, McMillan, Minder \& Soulsby 1984), to the T. parva stock (Mbogo, Kariuki, Nguni \& McHardy 1996) and to the cattle breed (Paling \& Geysen 1981) but there are no reports of the role of anaemia in the pathogenicity or virulence of $T$. parva.

The objective of this study was to confirm the decline of packed cell volume (PCV) in animals infected by $T$. parva Katete and to associate the magnitude of this decline with the severity of the clinical reaction. In addition, we investigated the association between the size of RBCs and severity and lethality of ECF infection and compared the diameter of infected and non-infected RBCs to evaluate whether there is a preference of $T$. parva merozoites for larger or smaller cells.

\section{MATERIALS AND METHODS}

\section{Animals}

A retrospective analysis was conducted using haematological data from $18 \mathrm{~T}$. parva susceptible Belgian Black and White dairy cattle aged approximately 10 months that had been used in T. parva experiments between 1996 and 2000. The animals (16 heifers and two steers) were housed and fed on hay and concentrates and water ad libitum. These experiments were performed under the control of the Ethical Committee for Experimental Animals of the Institute of Tropical Medicine of Antwerp (Belgian registration number LA 1100120) which guarantees that animals do not suffer unnecessarily.

The animals were inoculated with T. parva Katete stabilates derived from the same reference stock $\mathrm{V} 1$. This stock was isolated from cattle in Katete district of Eastern Province of Zambia in 1983 (Berkvens, Geysen \& Lynen 1989). Stabilates used and doses inoculated were administered randomly and independently of mean corpuscular volume (MCV) and PCV values. The stabilates were injected subcutaneously in front of the right parotid lymph node. Blood samples were collected from the external jugular vein in ethylene diaminotetraacetic acid (EDTA) tubes for PCV and MCV measurements. All samples were processed on the day of collection. Blood and lymph smears were prepared daily from Day 7. Theileria parva infections were confirmed by the presence of schizonts in the lymph node biopsies 7-20 days after inoculation. Classification of reactions was according to Morzaria, Irvin, Voigt \& Taracha (1987). Non-lethal reactions were recorded when infected animals (confirmed by the presence of schizonts) survived for more than 24 days without chemotherapy.

\section{Haematological examinations}

The PCV and the mean corpuscular volume (MCV) were measured directly using a Technicon $\mathrm{H} 1 \mathrm{E}$ haematology analyzer (Bayer, Tarrytown, NY) together with other haematological parameters. The corpuscular size is determined by direct measurement and not derived from PCV and red blood cell counts (Feldman, Zinkl \& Jain 2000). Additional examinations were carried out on blood smears to determine the mean diameters of infected and non-infected RBCs. Red blood cells were measured prior to detection of piroplasms in blood (Day 7 ), at $1 \%$ parasitaemia and when maximum parasitaemia was recorded. Measurements were done using a micrometer (Mahr 46EH Digital micrometer head, Germany) fitted on a microscope at x1000 magnification. Examinations were performed around the "tail" of the smears where the cells were evenly spread. Twenty circular RBCs were measured from 3-4 fields for slides obtained before parasitaemia. Following the detection of piroplasms, the sizes of all infected cells in a particular field were measured, provided that they contained a single piroplasm and were circular. Thereafter, four unparasitized RBCs with normal shape and nearest to an infected cell were measured and an average size calculated.

\section{Statistical analysis}

Data analysis was done in Stata SE/8.0 (StataCorp 2003). The PCV and MCV profiles were drawn using cubic splines (five cross-median knots). The PCV, MCV, parasitaemia and RBC diameters were analysed using mixed models with animals as random effect. To ensure normality of the response variables, PCV values were arcsine transformed (Osborne 2002) while RBC diameters were transformed using natural logarithms. Parasitaemia values were logarithm transformed. Lethality group (lethal or nonlethal), period of infection (Days 0-7 or Days 14-21) and the period $x$ lethality group interaction were used as categorical fixed effects for PCV and MCV 
regressions. Separate analyses were done on RBC diameters before and during parasitaemia using the lethality group, the presence of piroplasms and the interaction between these two as categorical fixed effects.

\section{RESULTS}

The animals that suffered lethal reactions died on average 20 days after infection (18-23 days) following a period of high fever and parasitosis. The reactions of all animals undergoing non-lethal infections were also characterized by fever and parasitosis. However, parasitaemia was significantly higher in lethal than non-lethal reactions $(P=0.004)$ in the period 14-21 days. The mean parasitaemia (proportion of RBC infected by a piroplasm) in the lethal group was 3.88 (1.54-9.77) while that of the nonlethal group was $0.51(0.18-1.41)$.

\section{Packed cell volume}

Packed cell volume profiles for animals with lethal and non-lethal reactions are shown in Fig. 1. In the early stage of infection (Days $0-7$ ) there was no significant difference in PCV values between animals with lethal and those with non-lethal reactions $(P=$ 0.915) (Fig. 3A). In both groups, the PCV was significantly lower in the later stage compared to the early stage $(P<0.001)$. However, the PCV in the later stage of infection (Days 14-21) decreased significantly more in lethal reactions $(P<0.001)$.

\section{Mean corpuscular volume}

The MCV profiles following $T$. parva infection are shown in Fig. 2. When the MCV values were compared in the early stage of infection, there was a statistically significant difference between animals that developed lethal reactions and those that did not $(P<0.001)$. Animals that had lethal infections had lower initial MCV values (smaller cells) than those that suffered non-lethal infections. The boxand-whisker plot in Fig. 3B further shows that animals that suffered lethal reactions had a lower median MCV value than those that had non-lethal reactions. Late in the infection, $\mathrm{MCV}$ values remained lower in animals with lethal reactions $(P<0.001)$.
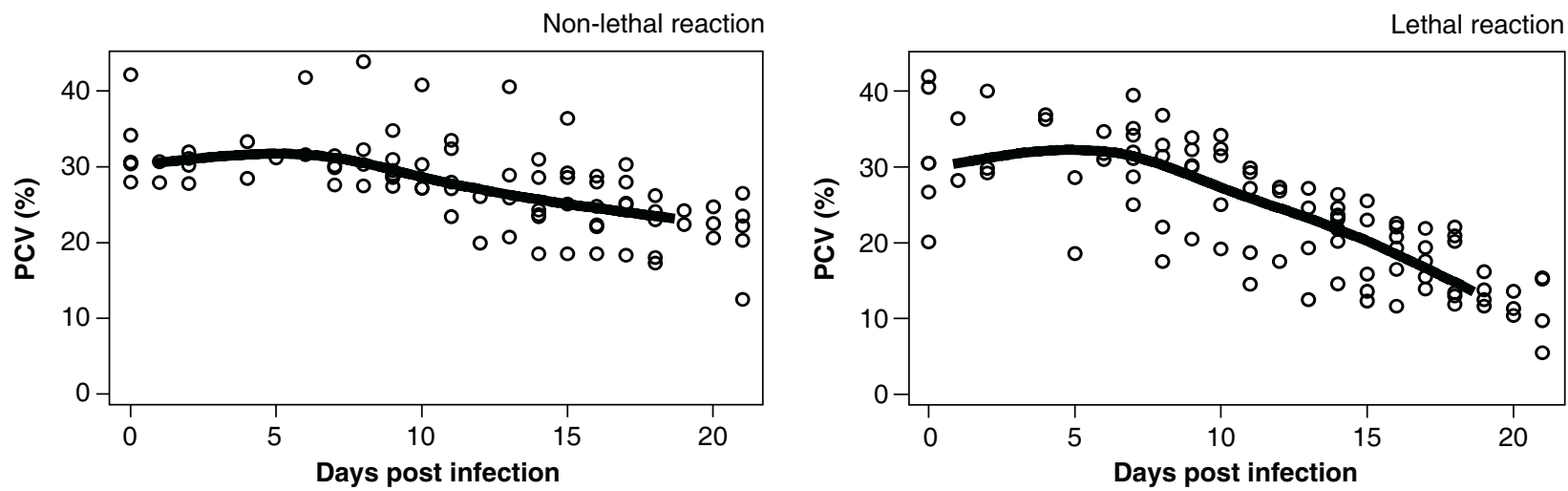

FIG. 1 Packed cell volume (PCV) as a function of days post infection in animals with non-lethal and lethal reactions. Open circles are individual observations and the solid line corresponds to a cubic spline function fitted through the observations
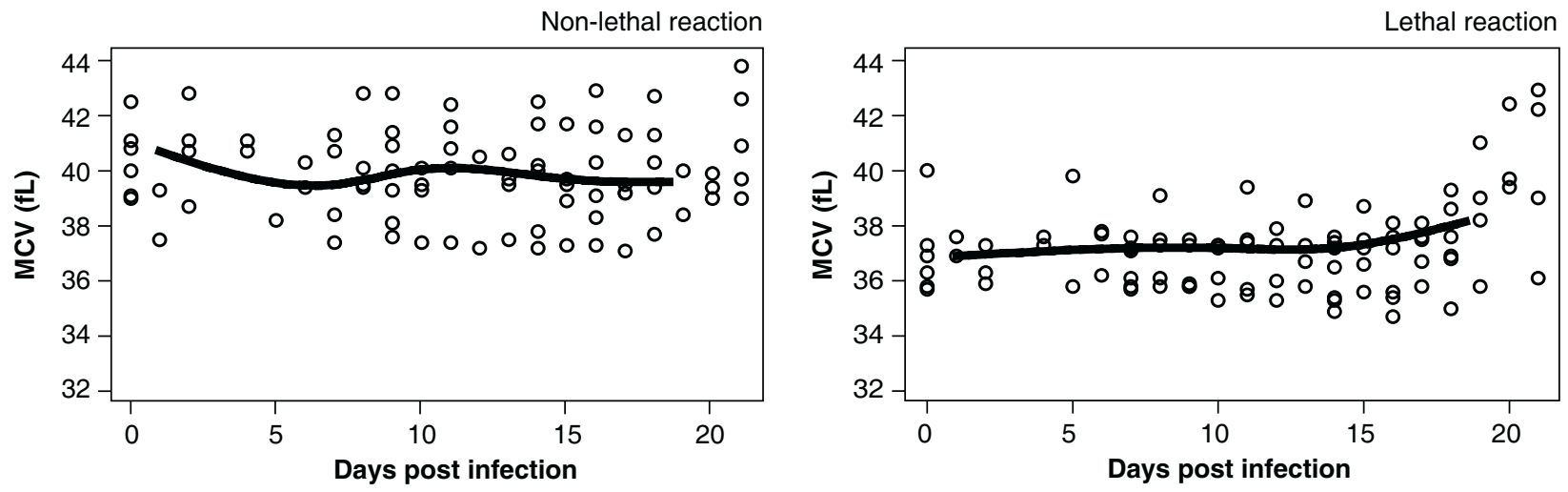

FIG. 2 Mean corpuscular volume (MCV) as a function of days post infection in animals with non-lethal and lethal reactions. Open circles are individual observations and the solid line corresponds to a cubic spline function fitted through the observations 
When plotting the initial frequency of RBC sizes in individual animals, the distribution of RBC sizes was wider in animals with non-lethal reactions (Fig. 4). While the lower limit of sizes was the same in both groups, the upper limit was lower in lethal T. parva infection.

\section{Sizes of parasitized and unparasitized erythrocytes in $T$. parva infections}

Animals that suffered lethal reactions had smaller RBC diameters before parasitaemia was detected $(P<0.001)$ (Table 1). In addition, for both lethal and non-lethal reactions, the diameter of parasitized

TABLE 1 Mean diameter (95\% confidence interval) of red blood cells $(\mu \mathrm{m})$

\begin{tabular}{|c|c|c|c|c|}
\hline \multirow{2}{*}{\multicolumn{3}{|c|}{ Before parasitaemia }} & Lethal & Non-lethal \\
\hline & & & $4.87(4.81-4.93)$ & $5.19(5.12-5.26)$ \\
\hline \multirow{2}{*}{ During parasitaemia } & Low parasitaemia & $\begin{array}{l}\text { Infected RBC } \\
\text { Non-infected RBC }\end{array}$ & $\begin{array}{l}4.46(4.41-4.51) \\
5.05(5.01-5.09)\end{array}$ & $\begin{array}{l}4.60(4.55-4.65) \\
5.21(5.17-5.25)\end{array}$ \\
\hline & Maximum parasitaemia & $\begin{array}{l}\text { Infected RBC } \\
\text { Non-infected RBC }\end{array}$ & $\begin{array}{l}4.45(4.40-4.51) \\
5.04(5.00-5.08)\end{array}$ & $\begin{array}{l}4.59(4.54-4.65) \\
5.20(5.16-5.24)\end{array}$ \\
\hline
\end{tabular}
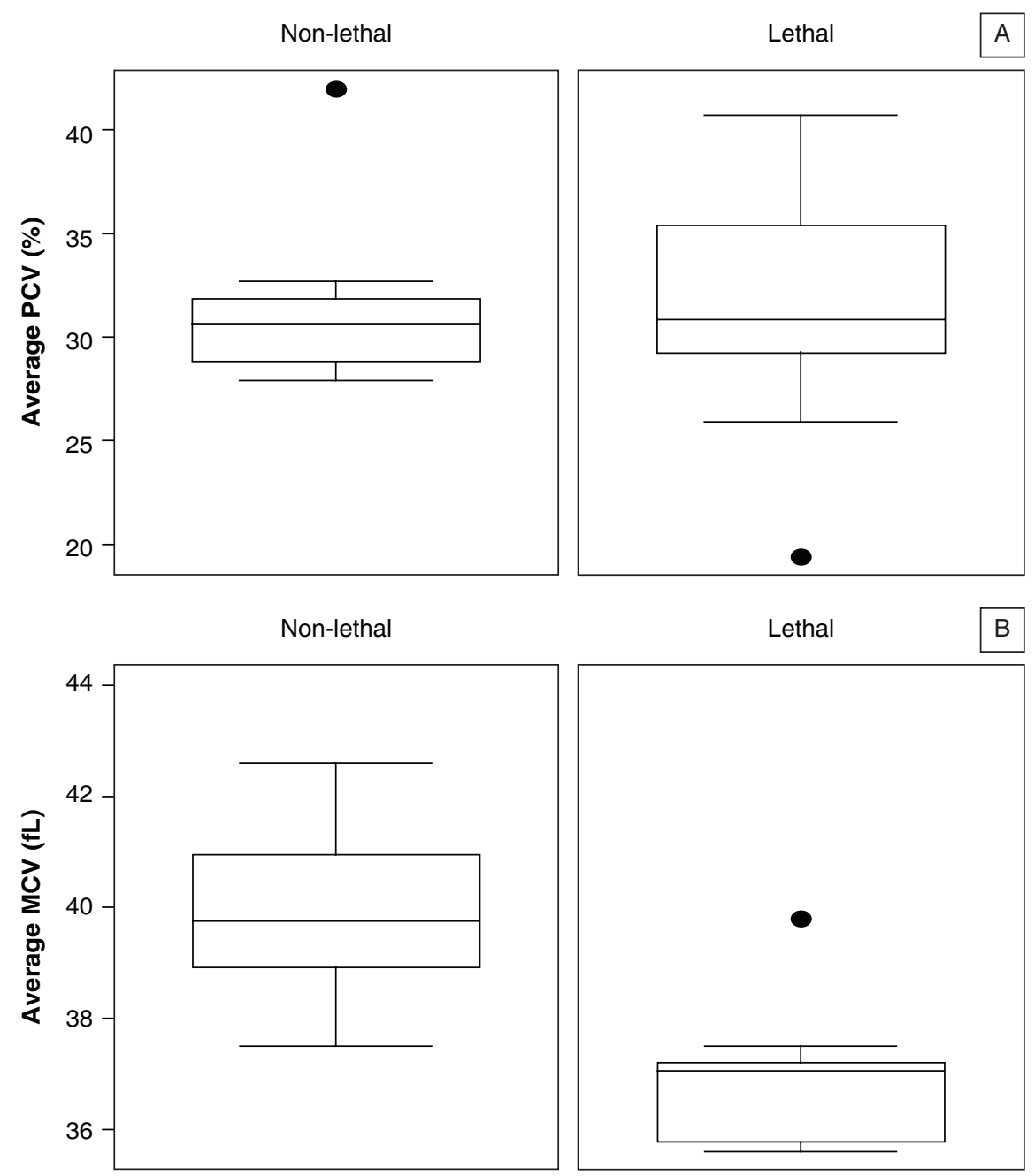

FIG. 3 The box-and-whisker plots of the average packed cell volume (PCV) and mean corpuscular volume (MCV) values for cattle infected with T. parva Katete in the first 7 days post infection. (A) PCV values and (B) MCV values. Dots represent outlier values. Boxes represent the $25^{\text {th }}, 50^{\text {th }}$ (median) and $75^{\text {th }}$ percentiles (including outliers). Whiskers include all observations except outliers 

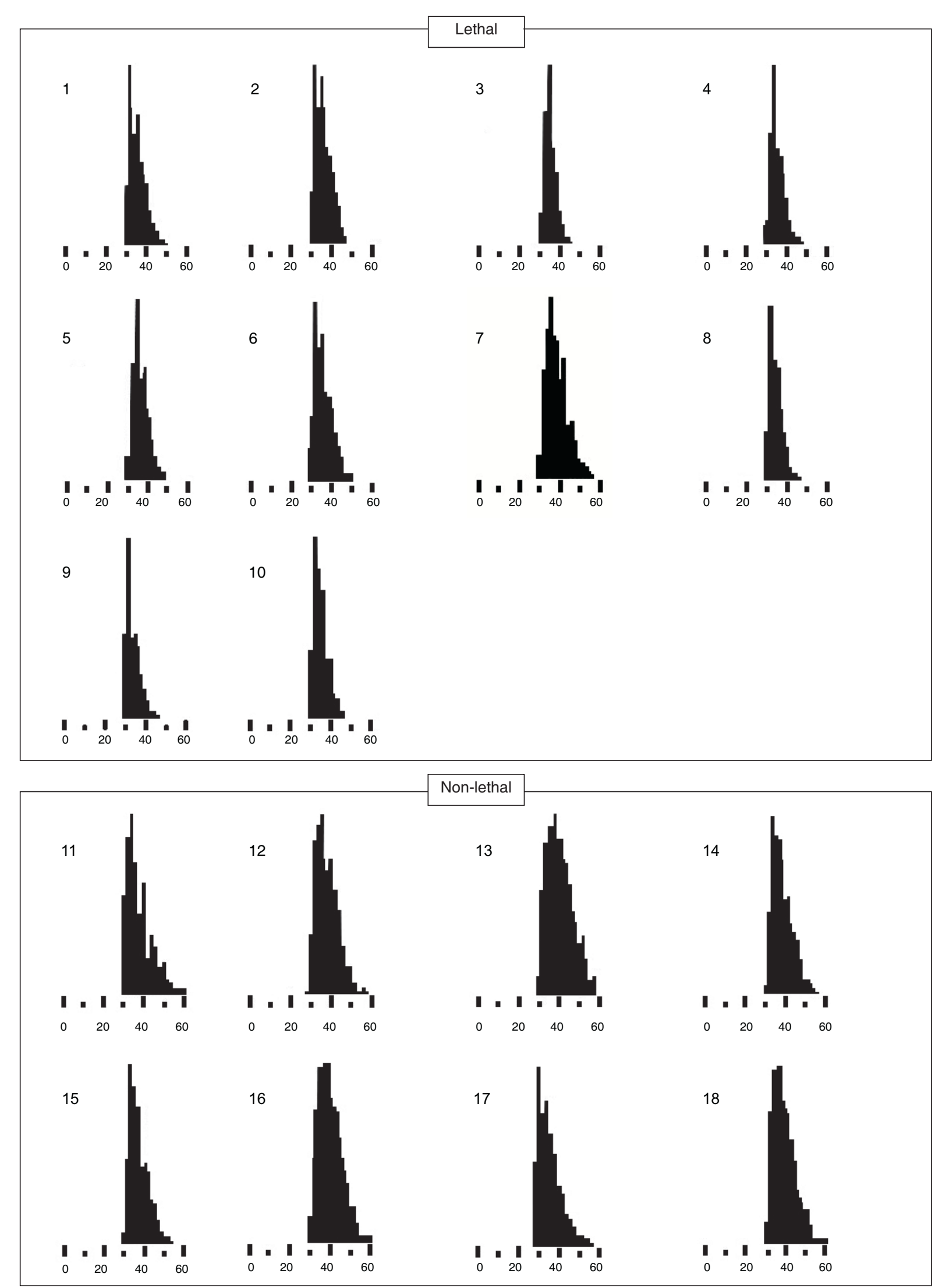

FIG. 4 Red blood cell volume (in fl) histograms from animals in the study taken in the first stage of infection (0-7 days) using a haematology analyzer. Animals $1-10$ had lethal reactions; $11-18$ had non-lethal reactions 
RBCs was significantly smaller than non-parasitized cells at low and maximum parasitaemia $(P<0.001)$. The average size of uninfected cells was significantly larger during the parasitaemia of lethal reactions than before.

\section{DISCUSSION}

This study confirmed that anaemia occurs in animals infected with the $T$. parva Katete stock. In the early stage of infection, the PCV profiles were similar in both outcome groups but the decrease in PCV was steeper in animals undergoing lethal reactions as the infection progressed.

Before Day 24 in both lethal and non-lethal infections, the MCV did not respond, presenting as a normocytic (non-regenerative) anaemia. This contrasts with our unpublished experimental observations with the T. parva Katete infections in Belgian Black and White cattle that during recovery following chemotherapy the MCV values increased, showing a regenerative response. It is possible that chemotherapy destroys most of the schizonts, halts lymphodestruction and allows the homeostatic mechanism to switch focus from a lymphoid to an erythroid response.

The other major finding was that animals that suffered lethal reactions had lower initial MCV values. Before infection, these animals were not anaemic as their PCV values were within normal range. No effect of age on MCV could be observed in this study as the number of observations was limited and the range of ages narrow. Birgel Junior, D'angelino, Benesi \& Birgel (2001) reported that PCV and RBC counts were significantly higher in younger Jersey cattle while MCV did not seem to be significantly affected by age. In contrast Penny, Scofield \& Cembrowicz (1966) working with different breeds (Friesian, Hereford, Shorthorn, Charolais, Guernsey and Ayrshire) with ages ranging from less that 2 years to over 10 years reported that PCV increased in bulls of ages 3-8 years compared other age groups while there was no significant difference in RBC and MCV values with age.

One possible explanation for the deaths in animals with low MCVs could be that there is an increased affinity of $T$. parva for small RBCs which would contribute to increased destruction or clearance of infected cells and subsequently to anaemia. This hypothesis is supported by the fact that uninfected red blood cells were larger during parasitaemia than before in lethal reactions in our study. Indeed, if the smallest cells of a pool become infected, the average size of the remaining cells will increase as observed.

Waugh, Narla, Jackson, Mueller, Suzuki \& Dale (1992) reported that aging RBCs lose both surface area and volume and are smaller than younger cells. Older (smaller) cells might be more easily infected by merozoites than younger (larger) cells. Shaw \& Tilney (1995) suggested that the deformability of the erythrocyte membrane may be a crucial factor in determining host cell susceptibility to invading $T$. parva merozoites. It may be that older cells lose some of the characteristics that prohibit the initial interaction with merozoites, making it easier for the parasite to enter the cell. Therefore, as parasitized cells are lysed or damaged by the parasite, or parasitized and non parasitized cells are coated with parasite antigen and removed by the immune system, animals with a pool of old cells may be more likely to develop anaemia than animals with a population of younger cells. It has been reported that following $T$. parva infections, there are considerable losses in parasitized RBCs and anaemic changes in animals with severe parasitaemia (Barnett 1957). Additionally, Shiono, Yagi, Kumar, Yamanaka \& Chikayama (2004) reported an increasing number of IgG bound red blood cells that were prone to removal by phagocytosis in animals infected with Theileria sergenti when a rise in parasitaemia was recorded. Otsuka, Yamasaki, Yamato \& Maede (2002) also reported immunological phagocytosis of IgG bound red blood cells in dogs infected with Babesia gibsoniwhich resulted in severe anaemia. Therefore, animals with a higher proportion of smaller cells may experience a higher fatality rate as parasite invasion of these fragile cells would lead to more severe anaemia. The anaemia may exacerbate the respiratory distress caused by pneumonia and oedema induced by the infiltration of the lungs with infected and dividing lymphoblasts (Irvin \& Morrison 1987) that is considered to be the cause of death in most T. parva infections (Irvin \& Mwamachi 1983).

The invasion of older RBCs observed in this study is in contrast to the findings of Mbassa et al. (1994) who reported that $T$. parva merozoites infected immature stages of erythrocytes. A possible explanation for this difference could be that Mbassa and colleagues were studying natural $T$. parva challenge in Tanzania and were thus looking at a different parasite and cattle with a different genetic make up.

These findings offer the possibility of predicting the possible outcome of $T$. parva infection based on an assessment of the initial MCV values and could 
contribute to improved diagnosis and control. It must be noted that MCV is only one additional parameter affecting the outcome of $T$. parva which is equally influenced by the parasite strain, the dose and cattle breed. Our observations pertain only to T. parva Katete in Belgian Black and White dairy animals and need to be extended to other breeds and other T. parva stocks. It might also have relevance for other blood parasites of animal health importance such as Babesia spp.

\section{ACKNOWLEDGEMENTS}

We express our sincere thanks to all members of staff of the Laboratory for Clinical Biology and the Department of Animal Health at the Institute of Tropical Medicine, Antwerp, Belgium for their assistance in looking after the experimental animals and processing the samples. This work was conducted within the framework of the Belgium Government funded project "Assistance to the Veterinary Services of Zambia (ASVEZA)".

\section{REFERENCES}

BARNETT, S.F. 1957. Theileriasis control. Bulletin of Epizootic Diseases of Africa, 5:343-357.

BERKVENS, D.L., GEYSEN, D.M. \& LYNEN, G.M. 1989. East Coast fever Immunisation in the Eastern Province of Zambia, in Theileriosis in Eastern, Central and Southern Africa, edited by T.T. Dolan. Proceedings of a Workshop on East Coast Fever Immunisation Held in Lilongwe, Malawi, 20-22 September 1988: 83-86.

BIRGEL, E.H. JUNIOR, D'ANGELINO, J.L., BENESI, F.J. \& BIRGEL, E.H. 2001. Reference values of the erythrogram of Jersey breed, raised in São Paulo state. Arquivo Brasileiro de Medicina Veterinária e Zootecnia, 15:1-9.

CONRAD, P.A., DENHAM, D. \& BROWN, C.G.D. 1986. Intraerythrocytic multiplication of Theileria parva in vitro: an ultrastructural study. International Journal for Parasitology, 16: 223-229.

DOLAN, T.T., YOUNG, A.S., LOSOS, G.J., McMILLAN, I., MINDER, C.H.E. \& SOULSBY, K. 1984. Dose dependent responses of cattle to Theileria parva stabilate. International Journal for Parasitology, 14:89-95.

FELDMAN, B.F., ZINKL, J.G. \& JAIN, N.C. 2000. Schalm's Veterinary Haematology, $5^{\text {th }}$ ed. Baltimore: Lippincott Williams \& Wilkins'.

IRVIN, A.D. \& MWAMACHI, D.M. 1983. Clinical and diagnostic features of East Coast fever (Theileria parva infection of cattle). Veterinary Record, 113:192-198.

IRVIN, A.D. \& MORRISON, W.I. 1987. Immunopathology, immunology and immunoprophylaxis of Theileria infections, in $I \mathrm{~m}$ mune responses in parasitic infections: immunology, immunopathology and immunoprophylaxis, Vol. III, edited by E.J.L. Soulsby. Boca Raton: CRC Press, Inc.
JURA, W.G.Z. \& LOSOS, G.J. 1980. A comparative study of the diseases in cattle caused by Theileria lawrencei and Theileria parva. 1. Clinical signs and parasitological observations. Veterinary Parasitology, 7:275-286.

MAXIE, M.G., DOLAN, T.T., JURA, W.G.Z., TABEL, H. \& FLOWERS, M.J.A. 1982. A comparative study of the diseases in cattle caused by Theileria parva or T. lawrencei, II. Haematology, clinical chemistry, coagulation studies and complement. Veterinary Parasitology, 10:1-19.

MBASSA, G.K., BALEMBA, O., MASELLE, R.M. \& MWAGA, N.V. 1994. Severe anaemia due to haematopoietic precursor cell destruction in field cases of East Coast fever in Tanzania. Veterinary Parasitology, 52:243-256.

MBOGO, S.K., KARIUKI, D.P., NGUNI, P.N. \& McHARDY, N. 1996. A mild Theileria parva parasite with potential for immunisation against East Coast fever. Veterinary Parasitology, 61:41-47.

MORZARIA, S.P., IRVIN, A.D., VOIGT, W.P. \& TARACHA, E. L.N. 1987. Effect of timing and intensity of challenge following immunisation against East Coast fever. Veterinary Parasitology, 26:29-41.

NORVAL, R.A.I., PERRY, B.D. \& YOUNG, A.S. 1992. The epidemiology of theileriosis in Africa. London: Academic Press.

OSBORNE, W.J. 2002. Notes on the use of data transformations. Practical Assessment, Research and Evaluation, 8:6.

OTSUKA, Y., YAMASAKI, M., YAMATO, O. \& MAEDE, Y. 2002. The effect of macrophages on the erythrocyte oxidative damage and the pathogenesis of anaemia in Babesia gibsoni-infected dogs with low parasitaemia. Journal of Veterinary Medical Science, 64:221-226.

PALING, R.W. \& GEYSEN, D. 1981. Observations on Rwandan strains of Theileria parva and the value of Theileria parva (Nyakizu) as a possible vaccine strain, in Advances in the control of theileriosis, edited by A.D. Irvin, M.P. Cunningham \& A.S. Young. Proceedings of an International Conference Held at ILRAD, Nairobi, 9-13 February 1981. The Hague: Martinus Nijhoff Publishers: 238-241.

PENNY, R.H.C., SCOFIELD, A.M. \& CEMBROWICZ, H. 1966. Haematological values for the clinically normal bull. British Veterinary Journal, 122:239-247.

RADLEY, D.E., BROWN, C.G.D., BURRIDGE, M.J., CUNNINGHAM, M.P., PEIRCE, M.A. \& PURNELL, R.E. 1974. East Coast fever: quantitative studies of Theileria parva in cattle. Experimental Parasitology, 36:278-287.

SHAW, M.K. \& TILNEY, L.G. 1995. The entry of Theileria parva merozoites into bovine erythrocytes occurs by a process similar to sporozoite invasion of lymphocytes. Parasitology, 111: 455-461.

SHIONO, H., YAGI, Y., KUMAR, A., YAMANAKA, M. \& CHIKAYAMA, Y. 2004. Accelerated binding of autoantibody to red blood cells with increasing anaemia in cattle experimentally infected with Theileria sergenti. Journal of Veterinary Medicine, B51:39-42.

WAUGH, R.E., NARLA, M., JACKSON, C.W., MUELLER, T.J., SUZUKI, T. \& DALE, G.L. 1992. Rheologic properties of senescent erythrocytes: loss of surface area and volume with red blood cell age. Blood, 79:1351-1358.

YOUNG, A.S., PURNELL, R.E., PAYNE, R.C., BROWN, C.G.D. \& KANHAI, G.K. 1978. Studies on the transmission and course of infection of a Kenyan strain of Theileria mutans. Parasitology, 67:99-115. 